\title{
ON THE UNIQUENESS OF THE QUASIHOMOGENEITY
}

\author{
PIOTR JAWORSKI \\ Institute of Mathematics, Warsaw University \\ Banacha 2, 02-097 Warszawa, Poland \\ E-mail: jwptxa@mimuw.edu.pl
}

Abstract. The aim of this paper is to show that the quasihomogeneity of a quasihomogeneous germ with an isolated singularity uniquely extends to the base of its analytic miniversal deformation.

1. Notation. Let

$$
f:\left(\mathbb{C}^{n}, 0\right) \rightarrow(\mathbb{C}, 0)
$$

be a germ of an analytic function and $\alpha_{1}, \ldots, \alpha_{n}, d$ be positive integers. $f$ is called quasihomogeneous (or weighted homogeneous) of type $\alpha=\left(\alpha_{1}, \ldots, \alpha_{n}\right)$ of quasidegree $d$, if

$$
\forall t \in \mathbb{C} \quad f\left(t^{\alpha_{1}} x_{1}, \ldots, t^{\alpha_{n}} x_{n}\right)=t^{d} f\left(x_{1}, \ldots, x_{n}\right) .
$$

The above can be stated more geometrically. $f$ is quasihomogeneous if it is equivariant under the $\mathbb{C}^{*}$ action on $\mathbb{C}^{n}$

$$
\begin{gathered}
\Psi: \mathbb{C}^{*} \times \mathbb{C}^{n} \rightarrow \mathbb{C}^{n}, \\
\Psi(t, x)=\left(t^{\alpha_{1}} x_{1}, \ldots, t^{\alpha_{n}} x_{n}\right), \\
f(\Psi(t, x))=t^{d} f(x) .
\end{gathered}
$$

If furthermore $f$ has an isolated singularity at the origin then its Milnor number $\mu$ is finite and there is a basis of the local algebra $\mathcal{O}_{n} / \mathcal{I}_{f}$ consisting of $\mu$ monomials $e_{1}, \ldots, e_{\mu}$, i.e.

$$
\mathcal{O}_{n}=\mathcal{I}_{f} \oplus \operatorname{Lin}_{\mathbb{C}}\left\{e_{1}, \ldots e_{\mu}\right\} .
$$

We remark that $\mathcal{I}_{f}$ denotes the gradient ideal of $f$,

$$
\mathcal{I}_{f}=\mathcal{O}_{n}\left(\frac{\partial f}{\partial x_{1}}, \ldots, \frac{\partial f}{\partial x_{n}}\right) .
$$

1991 Mathematics Subject Classification: Primary 32S30; Secondary 14 B07.

The paper is in final form and no version of it will be published elsewhere. 
Hence the germ at the origin of the following family is a miniversal deformation of $f$,

$$
F(x, \lambda)=f(x)+\sum_{j=1}^{\mu} \lambda_{j} e_{j} .
$$

Since $f$ is a polynomial in $x$, the domain of $F$ is $\mathbb{C}^{n} \times \mathbb{C}^{\mu}$. We shall call such deformation quasihomogeneous. The reason is that we can extend quasihomogeneity for $\mathbb{C}^{n} \times \mathbb{C}^{\mu}$ and make $F$ quasihomogeneous in both $x$ and $\lambda$.

We recall that

$$
\operatorname{qdeg} x_{1}^{a_{1}} \cdot \ldots \cdot x_{n}^{a_{n}}=\sum_{i=1}^{n} \alpha_{i} a_{i} .
$$

We introduce the weights for $\lambda$ 's by

$$
\beta_{j}=d-\operatorname{qdeg} e_{j} .
$$

Then

$$
\forall t \in \mathbb{C}^{*} \quad F\left(t^{\alpha_{1}} x_{1}, \ldots, t^{\alpha_{n}} x_{n}, t^{\beta_{1}} \lambda_{1}, \ldots, t^{\beta_{\mu}} \lambda_{\mu}\right)=t^{d} F(x, \lambda) .
$$

Moreover the $\mathbb{C}^{*}$ action on $\mathbb{C}^{\mu}$ preserves the discriminant of $F$ and its canonical stratification.

We remark that some $\beta$ 's may be negative or 0 . Thus, in the contrary to the standard quasihomogeneity, in the extended case the origin may not belong to the closures of all other orbits.

We shall show how to define this action for any miniversal deformation. First we establish notation. Let

$$
\widetilde{\Psi}: \mathbb{C}^{*} \times \mathbb{C}^{n} \times \mathbb{C}^{\mu} \rightarrow \mathbb{C}^{n} \times \mathbb{C}^{\mu},
$$

be the above extended $\mathbb{C}^{*}$ action;

$$
\widetilde{\Psi}(t, x, \lambda)=(\Psi(t, x), \psi(t, \lambda))=\left(t^{\alpha_{1}} x_{1}, \ldots, t^{\alpha_{n}} x_{n}, t^{\beta_{1}} \lambda_{1}, \ldots, t^{\beta_{\mu}} \lambda_{\mu}\right) .
$$

Obviously

$$
F(\widetilde{\Psi}(t, x, \lambda))=t^{d} F(x, \lambda)
$$

Let $G(y, \gamma)$ be another miniversal deformation of $f(G(y, 0)=f(y))$. Locally we have

$$
G(y, \gamma)=F(\widetilde{H}(y, \gamma))
$$

where $\widetilde{H}$ is a diffeomorphism

$$
\begin{gathered}
\widetilde{H}(y, \gamma)=\left(H_{\gamma}(y), h(\gamma)\right), \\
\widetilde{H}^{-1}(x, \lambda)=\left(H_{h^{-1}(\lambda)}^{-1}(x), h^{-1}(\lambda)\right) .
\end{gathered}
$$

We remark that the diffeomorphism $H_{0}(\cdot)$ preserves $f$ :

$$
f\left(H_{0}(y)\right)=f(y) .
$$

We define the $\mathbb{C}^{*}$ action on the neighbourhood of the origin of the domain of $G$ by

$$
\begin{array}{r}
\widetilde{\Phi}(t, y, \gamma)=\widetilde{H}^{-1}(\widetilde{\Psi}(t, \widetilde{H}(y, \gamma)))=\widetilde{H}^{-1}\left(\widetilde{\Psi}\left(t, H_{\gamma}(y), h(\gamma)\right)\right) \\
=\widetilde{H}^{-1}\left(\Psi\left(t, H_{\gamma}(y)\right), \psi(t, h(\gamma))\right)=\left(H_{h^{-1}(\psi(t, h(\gamma)))}^{-1}\left(\Psi\left(t, H_{\gamma}(y)\right)\right), h^{-1}(\psi(t, h(\gamma)))\right) \\
=(\Phi(t, y, \gamma), \varphi(t, \gamma)) .
\end{array}
$$


DeFinition 1. We call $\varphi$ defined by

$$
\varphi(t, \gamma)=h^{-1}(\psi(t, h(\gamma)))
$$

the induced $\mathbb{C}^{*}$ action.

Now we can state our main result.

TheOREM 1. Locally any two induced $\mathbb{C}^{*}$ actions $\varphi_{1}$ and $\varphi_{2}$ on the base of the same miniversal deformation $G$ coincide on some neighbourhood of the origin, i.e.

$$
\exists \delta_{1}, \delta_{2}>0 \quad \exists d_{1}, d_{2} \in \mathbb{N} \quad \forall t, \gamma \quad|t-1|<\delta_{1},\|\gamma\|<\delta_{2} \Longrightarrow \varphi_{1}\left(t^{d_{2}}, \gamma\right)=\varphi_{2}\left(t^{d_{1}}, \gamma\right)
$$

Remark. As $d_{1}$ and $d_{2}$ we may take the quasidegrees of $f$ in both underlying quasihomogeneities divided by a common factor.

2. The Euler vector fields. Crucial for us is the notion of an Euler vector field. For a given quasihomogeneous deformation $F(x, \lambda)$ we have

$$
\xi=\sum\left(\operatorname{qdeg} x_{i}\right) x_{i} \frac{\partial}{\partial x_{i}}+\sum\left(\operatorname{qdeg} \lambda_{j}\right) \lambda_{j} \frac{\partial}{\partial \lambda_{j}} .
$$

We recall its basic properties.

LEMMA 1.

1. $\xi$ is tangent to the orbits of the $\mathbb{C}^{*}$ action;

2. $\xi(F)=d F$ where $d=\operatorname{qdeg} f$.

Proof. We have

$$
\begin{aligned}
\frac{\partial \widetilde{\Psi}}{\partial t}(t, x, \lambda)_{\mid t=1}= & \left(\alpha_{1} x_{1}, \ldots, \alpha_{n} x_{n}, \beta_{1} \lambda_{1}, \ldots, \beta_{\mu} \lambda_{\mu}\right) \\
& =\left(\left(\operatorname{qdeg} x_{1}\right) x_{1}, \ldots,\left(\operatorname{qdeg} x_{n}\right) x_{n},\left(\operatorname{qdeg} \lambda_{1}\right) \lambda_{1}, \ldots,\left(\operatorname{qdeg} \lambda_{\mu}\right) \lambda_{\mu}\right),
\end{aligned}
$$

which proves the first point. The second one follows from the fact that $F$ is a sum of monomials of the same quasidegree $d$ and for any monomial we have

$$
\xi\left(x_{1}^{a_{1}} \cdot \ldots \cdot \lambda_{\mu}^{b_{\mu}}\right)=\sum a_{i}\left(\operatorname{qdeg} x_{i}\right) x^{a} \lambda^{b}+\sum b_{j}\left(\operatorname{qdeg} \lambda_{j}\right) x^{a} \lambda^{b}=\left(\operatorname{qdeg} x^{a} \lambda^{b}\right) x^{a} \lambda^{b} .
$$

3. The case of quasihomogeneous deformations. Before proving Theorem 1 we investigate the case of quasihomogeneous miniversal deformations.

TheOREM 2. If $G(y, \gamma)$ is a quasihomogeneous miniversal deformation then locally on the base any induced $\mathbb{C}^{*}$ action coincides with the canonical one.

Proof. Let $d_{1}$ be the quasidegree of $G$ and $d$ the quasidegree of the deformation $F$ from which we induce the $\mathbb{C}^{*}$ action $\widetilde{\Phi}$. (For $F$ we keep the notation from Section 1 .)

Step 1. $G$ is $\widetilde{\Phi}$ invariant.

$G(\widetilde{\Phi}(t, Y, \gamma))=F(\widetilde{H}(\widetilde{\Phi}(t, Y, \gamma)))=F(\widetilde{\Psi}(t, H(y, \gamma)))=t^{d} F(H(y, \gamma))=t^{d} G(y, \gamma)$.

Step 2. The "base part" of the Euler vector field $\xi$ is tangent to the orbits of the induced action $\varphi$. 
Let $\eta$ be a vector field tangent to the orbits of $\widetilde{\Phi}$.

$$
\eta=\left(\frac{\partial \Phi}{\partial t}\right)_{\mid t=1} \cdot \frac{\partial}{\partial y}+\left(\frac{\partial \varphi}{\partial t}\right)_{\mid t=1} \cdot \frac{\partial}{\partial \gamma}
$$

The derivative of $G$ along $\eta$ is a multiple of $G$ itself. Indeed:

$$
\eta(G)=\left(\frac{\partial G \circ \widetilde{\Phi}}{\partial t}\right)_{\mid t=1}=\left(\frac{\partial t^{d} G}{\partial t}\right)_{\mid t=1}=d G .
$$

Therefore

$$
\begin{aligned}
0 & =\left(d \xi-d_{1} \eta\right)(G) \\
& =\sum\left(d\left(\operatorname{qdeg} y_{i}\right) y_{i}-d_{1}\left(\frac{\partial \Phi_{i}}{\partial t}\right)_{\mid t=1}\right) \cdot \frac{\partial G}{\partial y_{i}}+\sum\left(d\left(\operatorname{qdeg} \gamma_{j}\right) \gamma_{j}-d_{1}\left(\frac{\partial \varphi_{j}}{\partial t}\right)_{\mid t=1}\right) \cdot \frac{\partial G}{\partial \gamma_{j}} .
\end{aligned}
$$

Since $G$ is miniversal, for any fixed $\gamma$ close to 0 the derivatives $\partial G / \partial \gamma_{j}$ are linearly independent modulo the gradient ideal $\mathcal{I}_{G}$ (see [1], Section 5.10). Therefore

$$
d_{1}\left(\frac{\partial \varphi}{\partial t}\right)_{\mid t=1}=\left(d\left(\operatorname{qdeg} \gamma_{1}\right) \gamma_{1}, \ldots, d\left(\operatorname{qdeg} \gamma_{\mu}\right) \gamma_{\mu}\right) .
$$

Thus $\varphi$ coincide with the canonical $\mathbb{C}^{*}$ action.

4. Proof of Theorem 1. We show in this section how Theorem 1 follows from Theorem 2.

Let $F_{1}$ and $F_{2}$ be two quasihomogeneous miniversal deformations of $f$, and $\widetilde{\Psi}_{1}, \widetilde{\Psi}_{2}$ the corresponding $\mathbb{C}^{*}$ actions. Since $G$ is induced from both of them, we have

$$
G=F_{1} \circ \widetilde{H}_{1}=F_{2} \circ \widetilde{H}_{2} .
$$

Thus

From Theorem 2 we know that

$$
F_{1}=F_{2} \circ \widetilde{H}_{2} \circ \widetilde{H}_{1}^{-1} .
$$

$$
\widetilde{H}_{2} \circ \widetilde{H}_{1}^{-1} \circ \widetilde{\psi}_{2} \circ \widetilde{H}_{1} \circ \widetilde{H}_{2}^{-1}
$$

coincide with $\widetilde{\psi}_{1}$. Hence the both induced actions on the base of $G$ coincide:

$$
\widetilde{H}_{1}^{-1} \circ \widetilde{\psi}_{2} \circ \widetilde{H}_{1} \approx \widetilde{H}_{2}^{-1} \circ \widetilde{\psi}_{1} \circ \widetilde{H}_{2} .
$$

5. Appendix. The real case. In the real analytic category one obtains the same results as in the complex. Indeed, the complexification of the miniversal deformation is a miniversal deformation of a complexification of a germ. Furthermore the $\mathbb{C}^{*}$ action is a complexification of the $\mathbb{R}^{+}$action. Therefore the uniqueness in the real analytic case is a consequence of the uniqueness in the complex case.

But in the $C^{\infty}$ category the uniqueness theorem is not valid. The reason is due to the fact that the deformation may have complex conjugated critical points which in $C^{\infty}$ category are "out of the picture". We illustrate this by the simplest example of the singularity $A_{2}$.

ExAmPLE. Let $f(x)=x^{3}$, then its quasihomogeneous miniversal deformation has the form

$$
F(x, a, b)=x^{3}+a x+b .
$$


Let $r$ be a $C^{\infty}$ function flat at the origin,

$$
r(a)>0 \text { if } a>0 \text { and } r(a)=0 \text { if } a \leq 0 .
$$

We consider the slight $C^{\infty}$ deformation of the Euler vector field:

$$
\xi_{1}=x\left(1-\frac{r(a)}{3 x^{2}+a}\right) \frac{\partial}{\partial x}+(2 a+r(a)) \frac{\partial}{\partial a}+3 b \frac{\partial}{\partial b} .
$$

We observe that its "base part" does not depend on $x$ and

$$
\xi_{1}(F(x, a, b))=3 F(x, a, b) .
$$

But, since the base parts of $\xi_{1}$ and of the Euler vector field are not parallel they define different $\mathbb{R}^{+}$actions on the base.

\section{References}

[1] V. I. Arnol'd, S. M. Guseřn-Zade, A. N. Varchenko, Singularities of Differentiable Maps I, Monogr. Math. 82, Birkhäuser, Boston, 1985. 